\title{
Exclusión social y políiticas de inclusión. Algunos elementos sobre la realidad europea y española
}

Joan Subirats, Maria Giménez e Anna Obradors

\section{Introducción}

En Europa, bajo el paraguas de un nuevo mundo globalizado, se están dejando atrás los principales parámetros socioeconómicos y culturales que rigieron la sociedad y su desarrollo durante años. Somos ahora testigos de un conjunto de profundas transformaciones que, velozmente, se suceden en todos los campos de la vida social.

En el campo de la producción, las viejas coordenadas del industrialismo se han visto transformadas por los impactos que han ocasionado los grandes cambios tecnológicos. Así, la llamada globalización económica, construida sobre la base de la revolución en los sistemas de información, ha permitido avanzar hacia un mercado mundial, en el que las distancias cuentan cada vez menos, y dónde el aprovechamiento de los costes diferenciales a escala planetaria ha desarticulado empresas y plantas de producción. En el mercado de trabajo, la temporalidad, el empleo a tiempo parcial o la flexibilidad han reemplazado a la especialización y 
la estabilidad, afectando principalmente a la calidad del empleo. En otras palabras: mientras el capital se ha hecho global y permanentemente movilizable, el trabajo continúa siendo local, cada vez menos permanente y más sujeto a la condición volátil de los espacios productivos. Por otra parte, los patronos de la sociedad de consumo se han instituido plenamente, contribuyendo a un ensanchamiento de la complejidad en el campo de las desigualdades y la exclusión social.

Desde el punto de vista social, las estructuras relativamente estables y previsibles del industrialismo han sufrido una acelerada transición hacia una realidad mucho más compleja caracterizada por una multiplicidad de ejes cambiantes de desigualdad. Aunque es cierto que en determinados segmentos entre los que anteriormente sólo existía continuidad de carencia han aparecido nuevas posibilidades de ascenso y movilidad social, también hallamos, por otro lado, nuevos e inéditos espacios de pobreza y de dificultad en el sobrevivir diario. Los riesgos de pobreza y vulnerabilidad social se han multiplicado y democratizado, castigando cada vez más personas y grupos sociales (BECK, 1998). Así, frente a la anterior estructura social de grandes agregados y de importantes continuidades, tenemos hoy un mosaico cada vez más fragmentado de situaciones de fracaso y de éxito generadas por una proliferación de riesgos y de interrogantes que, a su vez, provocan el surgimiento de fenómenos de búsqueda de certezas.

Desde el punto de vista de las relaciones de género y la familia, los cambios no son menores. El ámbito de convivencia primaria no presenta ya el mismo aspecto que tenía en la época industrial. El escenario es hoy muy distinto. La equiparación formativa entre hombres y mujeres es muy alta aunque ello no se traduzca en una mejora en la posición social y de mercado de estas últimas. La incorporación de las mujeres al mercado de trabajo formal aumenta sin cesar, a pesar de las múltiples dificultades y discriminaciones que se mantienen. Junto a estos cambios, hay que tener en cuenta que los roles de género, tanto para hombres como para mujeres, apenas se han modificado. Así, crecen las tensiones por la doble jornada laboral de las mujeres y las dificultades de la conciliación domésticolaboral. En el caso de los hombres, las dificultades principales radican en la pérdida de su estatus como cabeza de familia y sustentador de la misma. Todo ello actúa en combinación con los cambios que se están produciendo en la forma y composición de los núcleos familiares que integran las sociedades europea y española dónde, en los últimos años, han aumentado sin cesar la cantidad de hogares unipersonales integrados por personas de más de 65 años - sobretodo entre las mujeres así como el número de hogares encabezados por una mujer sola con hijos (SAINSB URY, 1999).

Todo lo anterior además, se produce en un contexto general de crisis y desvanecimiento del Estado de Bienestar en Europa. Así, el conjunto de cambios y de profundas transformaciones en las esferas productiva, social y familiar, no han encontrado una respuesta política clara y decidida. Con la irrupción del neoliberalismo, la fragmentación y la retirada institucional aumenta: surgen nuevas instituciones supraestatales y, en el campo de la política social se realizan importantes procesos de descentralización y focalización de las intervenciones. Los procesos de toma de decisiones se basan cada vez más en lógicas de interdependencia, de capacidad de influencia y de poder relacional, 
que en un estatuto orgánico o en el ejercicio de jerarquías formales (KICKERT, 1997). En toda Europa además, la introducción progresiva de procesos de casi-mercado y de gerencias privadas en los servicios públicos locales son prácticas en clara ascendencia.

\section{El concepto de exclusión social}

Durante los años 70s, en muchos países de la Unión Europea se había asumido que en estos países la pobreza material había sido reducida a un "estado residual de acontecimientos que desaparecería con el progreso y el crecimiento" (Comisión EuropeA, 1992, p.7). Sin embargo, actualmente, más allá del plano puramente económico, asistimos a una ampliación progresiva de las desigualdades sociales, y la emergencia de nuevas fracturas que cada vez desplazan a más individuos y grupos hacia la precariedad económica y social. Es por ello que el concepto más clásico de pobreza, relativo a la escasez o ausencia de recursos materiales, debe ser reconsiderado ante la noción de exclusión social, que brinda la posibilidad de desarrollar análisis más complejos, capaces de dar cuenta tanto de las viejas y persistentes desigualdades sociales, como de las nuevas fracturas.

En España igual que en Europa, la investigación alrededor de la exclusión social se inició y se mantiene por la posibilidad que ofrece este concepto de abordar globalmente nuevas y viejas desigualdades enraizadas en lo más profundo de las estructuras social y económica de unos países que, como hemos señalado, se hallan inmersos en un intenso proceso de cambios en distintos niveles. Más allá de la pobreza, en este contexto generalizado de cambios, emergen otros ejes, más trasversales, que operan como espesos filtros a la inclusión social. Así, las viejas formas de desigualdad de renta, sumadas a las brechas de ciudadanía, a la pérdida de vínculos y redes sociales, u otros, otorgan pleno sentido al uso cada vez más extendido al rico y complejo concepto de exclusión social.

En el plano empírico, la exclusión social puede entenderse como una situación concreta fruto de un proceso dinámico de acumulación, superposición y/o combinación de diversos factores de desventaja o vulnerabilidad social, y que genera entre las personas o grupos sociales, una situación de imposibilidad o dificultad intensa de acceder a los mecanismos de desarrollo personal, de inserción socio-comunitaria y a los sistemas preestablecidos de protección social. Por otra parte, y en relación con lo anterior, el concepto de vulnerabilidad social delimita toda una serie de situaciones que se hallan presididas por un equilibrio social precario que puede verse transformado en exclusión social mediante un proceso de intensificación de uno o más factores de exclusión, o con la aparición de otros nuevos, que pueden o no estar relacionados con el resto.

Así pues, la exclusión social implica la acumulación de factores que además, pueden presentarse con intensidades y combinaciones variables. Es decir, la precariedad laboral, por ejemplo, puede comprender desde el trabajo no cualificado a tiempo parcial hasta el trabajo sin derechos ni garantías sociales en el espacio de la economía sumergida. A su vez, existen combinaciones de factores que pueden acelerar procesos o sostener situaciones de las cuales resulta muy difícil salir. Así, una situación de violencia familiar que suponga una pérdida de lazos o redes sociales, unida al desempleo, a la adicción, o la pobreza económica etc. puede abrir procesos de no retorno hacia la soledad, la enfermedad y en definitiva, la exclusión social. 
Desde este punto de vista, la exclusión social es un fenómeno altamente complejo en la medida en que hace referencia a una multidimensionalidad y una multifactorialidad que operan de forma dinámica según los contextos y que poseen sus efectos, más súbitos o dilatados, en un espacio de tiempo determinado. Las dimensiones y factores de exclusión se presentan en todas las esferas del desarrollo personal y social. Por tanto, podemos observar factores tan dispares como la irregularidad administrativa a la que se hallan sometidos muchos inmigrados, la violencia intrafamiliar, el trabajo precario, el analfabetismo, etc. Así pues, a lo largo de la vida de una persona pueden irse acumulando una serie de factores que, en su combinación y mutua influencia, pueden conducirle hacia un escenario particular de dificultades y exclusión social. De la misma manera, tal y como postula Castel (1995), la clase media, la gran protegida por el sistema social del bienestar, se torna especialmente vulnerable a las dinámicas del cambio social. Ya no son sólo unos pocos, sino que el riesgo y vulnerabilidad social se expande pudiendo afectar a las posiciones centrales - y no sólo a las marginales - de la estructura social. Así, aparecen posibilidades de entrada y salida del sistema de una proporción cada vez mayor de la sociedad.

La exclusión social es susceptible de ser abordada desde los valores, desde la acción colectiva, desde la práctica institucional y desde las políticas públicas. El territorio, geográficamente delimitado, (Estado Español, comunidades autónomas, municipios, barrios...) es el marco donde se entreteje la realidad social con la política pública. Esta es la unidad básica de análisis para poder describir y comprender cuáles son y cómo operan los factores generadores de la exclusión social y, por otro lado, cómo se articulan las oportunidades para la inclusión. Los elementos más territoriales de la exclusión social (la configuración socio-espacial del territorio, la articulación político-administrativa, las formas existentes en lo relacional y, en definitiva, el régimen local de bienestar) no inciden tanto en la definición de los ejes de integración social y de los factores de exclusión, como en la dinámica que se establezca entre ellos.

\section{La exclusión social en la agenda de la Unión Europea}

La Unión Europea, en la cumbre de Lisboa del año 2000, destacó que la exclusión social no podía considerarse simplemente como inevitable o como un efecto indeseable del desarrollo económico. Desde las instancias europeas, con el objetivo de mantener el modelo de crecimiento europeo basado, teóricamente, en el desarrollo económico, la equidad y la inclusión social, se decidió impulsar una serie de Planes Nacionales de Inclusión Social. De esta manera, se trasladaba a los países miembros la responsabilidad de que, de forma complementaria a sus propias políticas, diseñasen y aprobasen unos Planes Nacionales que mostrasen de forma integrada sus respuestas a los retos de la cohesión social. Desde la cumbre de Lisboa se llevan aprobados dos planes en cada país de los 15 de la Unión Europea, y se ha ido siguiendo un proceso de convergencia entre los mismos y las iniciativas de la Comisión, en aplicación del llamado "método abierto de coordinación".

Los objetivos que marcó la estructura de los Planes Nacionales impulsados desde la Unión Europea fueron los de acceso al empleo y recursos, derechos, bienes y servicios de todos los ciudadanos; la prevención de los riesgos de exclusión; 
la actuación a favor de los más vulnerables y la movilización de todos los agentes sociales en todos los ámbitos y propuestas de cada Plan.

Las recomendaciones de la UE apuntaban a que la problemática de la pobreza y la exclusión social debía estar inscrita en el conjunto de ámbitos políticos. $\mathrm{Al}$ mismo tiempo se recomendaba que se reconociera la importancia de la dimensión local y regional, y que se avanzara en un enfoque integrado y estratégico, que incluyera una perspectiva transversal que se consideraba esencial para afrontar las situaciones de exclusión. Se insistía en que se tenían que definir y desarrollar respuestas políticas para ayudar a las personas más marginalizadas y excluidas, integrando mejor los ámbitos de la salud o la cultura en los restantes ámbitos de actuación. La UE insistió también en que los Planes Nacionales incorporaran la dimensión de igualdad entre las mujeres y los hombres en cada etapa de los planes, en la definición de los retos, en la elaboración, aplicación y evaluación de las políticas, en la selección de indicadores y objetivos y en la participación de los interesados.

En estos momentos estamos en pleno proceso de implementación y seguimiento de los quince planes aprobados por otros tantos países de la Unión Europea en el año 2003, con vigencia hasta el 2005, que representan la segunda oleada de planes desde la cumbre de Lisboa.

El análisis del conjunto de planes nacionales, refleja, desde nuestro punto de vista, que tanto los conceptos que se utilizan para definir la exclusión social, como las líneas de respuesta que se apuntan son muy distintas de país a país. Por ello, no nos puede extrañar que el resultado final sea un conjunto de planes que si bien se nos presentan con formatos semejantes y dentro de una misma perspectiva europea, en realidad son sustancialmente diferentes tanto a nivel cuantitativo como cualitativo.

Si los analizamos desde un punto cualitativo, lo cierto es que no hay muchas diferencias sobre los colectivos o sobre los ámbitos en los que se pretende actuar. Las diferencias surgen cuando examinamos su nivel de concreción y el grado de transversalidad de las medidas que proponen. Y, en este sentido, es significativo reseñar que las tradiciones de políticas de bienestar de

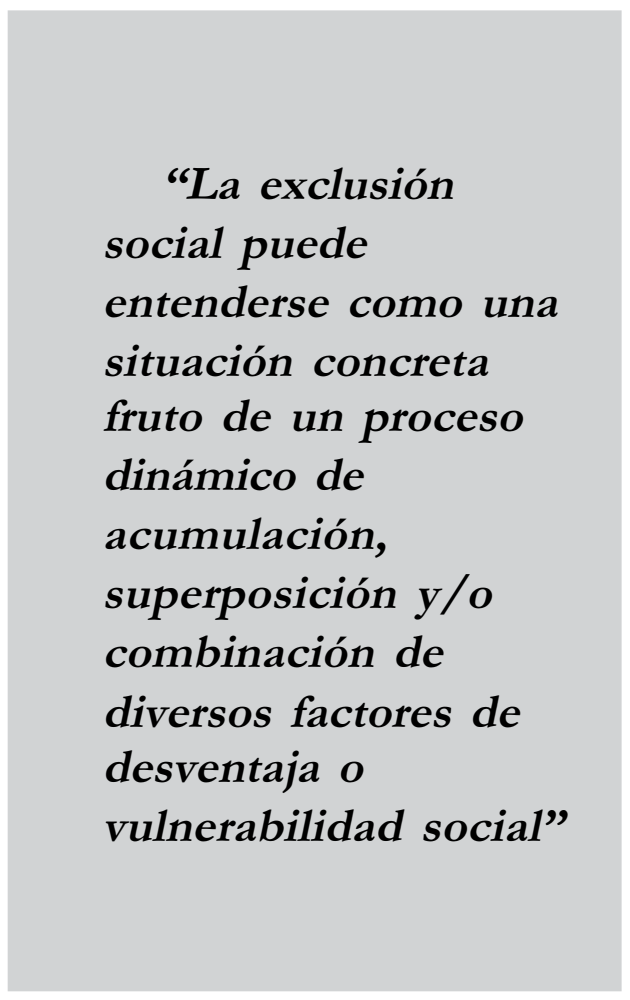

cada país, sus distintos y bien conocidos puntos de partida y sus niveles de gasto social, poco nos indican en relación a la "calidad" intrínseca de los Planes presentados. Lo cual apunta a que estamos tratando de temas relativamente nuevos, sobre los que los estados, al margen de su trayectoria histórica, no disponen de mucha experiencia acumulada, lo que permite que países de incorporación tardía a las políticas de bienestar, presenten planes de 
relativo calado, mientras que países con políticas de bienestar bien enraizadas, aparezcan con deficiencias significativas en sus planteamientos.

No hemos de olvidar que hasta principios de los años ochenta, los países avanzados presentaban estructuras sociales relativamente homogéneas cruzadas por una lógica de estratificación muy clásica. Ello había comportado la consolidación histórica de un patrón de necesidades sociales uniformes, concentradas en determinados estratos, derivadas de la incapacidad del acceso a algunos bienes básicos, debido a rentas salariales insuficientes o falta coyuntural de trabajo. A esta configuración de necesidades, el modelo clásico de bienestar respondía con una oferta centralizada y estandarizada de servicios y transferencias, articulada básicamente en torno a sistemas nacionales de salud y a mecanismos de seguro social. Ahora, cuando los factores antes analizados han generado la nueva polarización inclusión/ exclusión, que se superpone al eje clásico de desigualdad vertical, el problema es que ninguno de los regímenes de bienestar europeos se encuentra bien equipado para enfrentar el problema de la exclusión social. En estos años se han tratado de hacer cosas en toda Europa frente a estos retos. Y los Planes Nacionales son un ejemplo de las potencialidades pero también de las complejidades y límites de lo que hasta ahora se ha hecho y se sigue haciendo.

\section{La situación en España}

Actualmente, en España, a pesar de los cambios, el sistema productivo parece continuar siendo el principal mecanismo de integración y, por lo tanto, también de exclusión social. Ello significa que en la mayor parte de procesos y situaciones de exclusión, la participación en el sistema de producción de mercado impone su lógica sobre el resto de ámbitos y espacios en que pueden desarrollarse factores de exclusión. Este mecanismo, en la medida en que determina la "utilidad" de las personas, socava uno de los principios básicos sobre los que se asienta la inclusión social: la igualdad.

A diferencia de lo que ocurre en otros países europeos, en España la intervención estatal no está haciendo lo suficiente para nivelar estos desequilibrios. Las personas que quedan excluidas del mercado productivo también quedan fuera o al margen del sistema de redistribución y/o de las políticas de reconocimiento de la diferencia. Es decir, de políticas inclusivas de ciudadanía.

El análisis que hemos realizado de la situación en España nos señala y confirma algunos de los elementos conceptuales o analíticos que hemos venido utilizando en este artículo. Si nos fijamos en las características personales de los sujetos más vulnerables, hemos comprobado que las personas que aparecen en mayor medida en situaciones de desvinculación o exclusión social, serían las mujeres, los ancianos y los hogares de adultos sin hijos. Y, en cambio, las personas que relativamente presentan una menor concentración de factores de vulnerabilidad serían los hombres, las personas comprendidas entre los 16 y 44 años, y los hogares compuestos por adultos con uno o más niños.

Si tratamos de ver de manera diferenciada cada uno de los factores de exclusión y sus relaciones con las distintas personas de la muestra que hemos utilizado para nuestro análisis, veremos que las mujeres cuando son jóvenes se ven muy afectadas por situaciones de desempleo sin protección alguna. En cambio, más adelante, al trabajar como "amas de casa", o realizar trabajos domésticos irregulares e informales su visibilidad como colectivo "desconectado" es menor, 
tanto en lo referente al tema del desempleo como en aspectos como enfermedad o discapacidad. Pero, diríamos que la pobreza severa esta muy feminizada, y son especialmente complicadas las situaciones de los hogares monomarentales.

Los hombres ven claramente agravada su situación cuando alcanzan edades avanzadas, y van perdiendo relaciones, aumentando su condición de dependientes. Diríamos que el hecho de vivir solos y/o de ser extranjeros agrava especialmente su situación.

Si nos referimos a la situación general, confirmaríamos que la exclusión social, en sus distintos componentes y factores desencadenantes, debería analizarse siempre viendo cómo afecta el género, la edad y la procedencia. Tres elementos que, desde nuestro punto de vista, operan como factores de intensificación de las diversas situaciones de vulnerabilidad y exclusión social generadas por otros ámbitos (económico, laboral, formativo, etc.)

Si vamos más allá de los factores personales, y queremos trazar algunos perfiles de los colectivos de la población española más vulnerables, veremos que el campo laboral sigue resultando muy determinante. Sea porqué no se logra entrar en el mismo, sea porque no se logra estabilidad alguna, sea porque sin trabajo no hay prestaciones sociales seguras. Y más allá de ello la ancianidad y la pobreza son rasgos colectivos muy claros. $\mathrm{Y}$ una vez más, género, edad o procedencia siguen siendo determinantes a la hora de explicar situaciones y carencias. No podemos dejar de reseñar el enorme peso que la tradición industrial otorgó al hecho de trabajar como pasaporte a las prestaciones derivadas de las políticas sociales. Si no dispones de buena for mación, si tus condiciones de salud están deterioradas o no tienes experiencia laboral acreditada (y en ella no sirve el trabajar ho- ras y horas en labores cuidadoras o domésticas), tus riesgos de exclusión aumentan exponencialmente. $\mathrm{Y}$ si no hablamos de trabajo, entonces la soledad o el analfabetismo son muy relevantes en el desencadenamiento de riesgos y situaciones de exclusión.

De lo que hemos mencionado, surge una fotografía aún borrosa, pero significativa de la exclusión social en España. Fotografía en la que encontramos mujeres cuyo trabajo no es reconocido, ancianos con problemas de soledad y pobreza, y emigrados que viven aislados y en condiciones de precariedad significativa. Pero más allá de esos perfiles y de los demás ya mencionados, sabemos de combinaciones múltiples y de factores que se superponen y que exigen una mayor complejidad y finura en las políticas de respuesta.

\section{Políticas públicas e inclusión social}

En términos generales, las políticas y acciones para la inclusión social se estructuran, y por tanto, pueden entenderse vinculadas al entorno de tres ejes fundamentales: igualdad, diferencia y autonomía. Estas tres dimensiones dan lugar a tres tipos básicos de políticas para la inclusión social:

- Políticas de redistribución de recursos que avanzan hacia la igualdad, recortando las desigualdades generadas por el sistema capitalista de mercado.

- Políticas de reconocimiento que permiten prestar atención a las diferencias en una sociedad cada vez más compleja y diversificada.

- Políticas de fortalecimiento relacional y de participación que facilitan el desarrollo de la vida cotidiana con autonomía.

En España nos hallamos ante un modelo de respuesta altamente familiarista y contributivo. Así, si hablábamos de los 
Población vulnerable en España a partir del panel de hogares de la UE (2000)

\begin{tabular}{|c|c|c|c|c|}
\hline & \multicolumn{2}{|c|}{$\begin{array}{l}\text { Población } \\
\text { vulnerable }\end{array}$} & \multicolumn{2}{|c|}{ Total de la muestra } \\
\hline \multicolumn{5}{|l|}{ Sexo } \\
\hline Hombre & $33,9 \%$ & 3.477 & $47,3 \%$ & 17.107 \\
\hline Mujer & $66,1 \%$ & 6.785 & $52,7 \%$ & 19.041 \\
\hline \multicolumn{5}{|l|}{ Grupos de edad } \\
\hline $16-29$ & $18,7 \%$ & 1.922 & $24,4 \%$ & 8.837 \\
\hline 30-44 años & $20,5 \%$ & 2.101 & $25,4 \%$ & 9.175 \\
\hline 45-64 años & $32,7 \%$ & 3.357 & $26,9 \%$ & 9.726 \\
\hline 65-99 años & $28,1 \%$ & 2.882 & $23,3 \%$ & 8.410 \\
\hline \multicolumn{5}{|l|}{ Tipo de hogar } \\
\hline Unipersonal menor de 65 años & $2,1 \%$ & 215 & $2,4 \%$ & 857 \\
\hline $\begin{array}{l}\text { Unipersonal mayor o igual } \\
\text { a } 65 \text { años }\end{array}$ & $4,2 \%$ & 430 & $4,2 \%$ & 1.528 \\
\hline 2 o más adultos sin niños ${ }^{1}$ & $53,7 \%$ & 5.507 & $45,8 \%$ & 16.548 \\
\hline 1 adulto con niños & $1,2 \%$ & 123 & $1,1 \%$ & 392 \\
\hline Varios adultos y 1-2 niños & $36 \%$ & 3.694 & $43 \%$ & 15.557 \\
\hline 2 adultos con 3 o más niños & $2,8 \%$ & 292 & $3,5 \%$ & 1.262 \\
\hline \multicolumn{5}{|l|}{ Lugar de nacimiento } \\
\hline España o CE & $98,3 \%$ & 10.063 & $98,8 \%$ & 35.561 \\
\hline Países anglosajones ${ }^{2}$ & & 4 & & 13 \\
\hline Resto del mundo & $1,7 \%$ & 172 & $1,2 \%$ & 432 \\
\hline Total & $100 \%$ & 10.262 & $100 \%$ & 36.148 \\
\hline \multicolumn{5}{|c|}{ Porcentajes por encima de la muestra total } \\
\hline \multicolumn{5}{|c|}{ Porcentajes por debajo de la muestra total } \\
\hline
\end{tabular}

Fuente: Subirats, J (Dir.) (2004); Pobreza y exclusión social. Un análisis de la realidad española y europea, Colección Estudios Sociales no 16, Fundación "La Caixa", Barcelona, p. 90

${ }^{1}$ Se ha considerado bajo la categoría "niño" ser menor de 16 años, o ser un joven con una edad comprendida entre los 16 y los 25 años, que se declare económicamente inactivo, o desempleado desanimado, o que trabaje menos de 15 horas semanales, y viva en el mismo hogar que el padre, la madre o ambos.

${ }^{2}$ Se incluyen en esta categoría nacidos en América del Norte, Australia y Oceanía.

cambios que se producen en el marco de la familia y las relaciones de género, la inexistencia de redes sociales y familiares de apoyo se convierte en un factor clave del análisis de la exclusión social. Ello es así porqué, por una parte, el peso del cuidado y atención a las personas sigue recayendo principalmente sobre las unidades familiares 
que se convierten en un soporte fundamental para la inclusión y el desarrollo personal. Por otra parte, la mayor parte de beneficios sociales se hallan estrechamente ligados a una cotización laboral previa. En este contexto, las políticas para la inclusión que se desarrollan, son más asistenciales que capacitadoras, y van más encaminadas a la atención a colectivos definidos a priori, que a la debilitación de los factores que se hallan en la base de les desigualdades.

Además, y a pesar de los esfuerzos y la voluntad de las familias y las redes sociales y comunitarias, estos agentes no siempre pueden llegar a articular los mecanismos de reciprocidad necesarios para dotar de autonomía los grupos sociales o personas más vulnerables o excluidas. En otras palabras, la familia y la red social - cuando existen - llegan a ser soportes de gran importancia, pero nunca llegan a corregir por sí solas las profundas fracturas impuestas por el mercado e insuficientemente paliadas por la acción pública y las instituciones.

Para combatir la exclusión social desde las políticas públicas, los planteamientos estratégicos y lo más integrales posible, son fundamentales. En la medida en que la exclusión social tiene un carácter estructural, las políticas públicas deben ser lo más estratégicas posible. Es decir, deben atacar las causas de la exclusión, y no solamente paliar superficialmente sus síntomas más evidentes. Desde nuestro punto de vista, las políticas de inclusión que se emprendan deberían priorizar la debilitación de los factores que generan la exclusión, la precariedad y la marginación social. Deberían por tanto, orientarse hacia procesos de promoción, prevención e inserción universalistas, fortaleciendo y reestableciendo los vínculos y las redes laborales, sociales, comunitarias y familiares de las personas. La orientación estratégica de la política social comporta emprender procesos de capacitación y mejora de la autonomía personal, huyendo de las lógicas meramente asistenciales y focalizadas.

Además, como hemos comentado en un inicio, las políticas públicas deben ser diseñadas desde planteamientos integrales, abarcando el conjunto de situaciones de desventaja que conducen a la exclusión social. Este requisito no es de fácil consecución si tenemos en cuenta la tendencia a sectorializar las políticas sociales

"En una fotografía de la exclusión social en España, encontramos mujeres cuyo trabajo no es reconocido, ancianos con problemas de soledad y pobreza, y emigrados que viven aislados y en condiciones de precariedad significativa"

que se mantiene en un Estado de Bienestar en claro retroceso.

Por otra parte, la fórmula operativa para alcanzar políticas estratégicas de inclusión pasa también por la articulación de redes de trabajo transversal y multinivel. Es decir, por poner en relación diferentes áreas y niveles administrativos de actuación que establezcan mecanismos de colaboración y trabajo conjunto aprovechando la experiencia y el conocimiento del tercer 
sector, y no delegando en él y/o en la empresa privada la gestión e implementación de las acciones e intervenciones.

El actual escenario de fragmentación institucional generada a partir de los procesos de descentralización ascendente (UE), descendente (Comunidades Autónomas y municipios) y lateral (partenariados públicos-privados, externalización de servicios y presencia de ONGs) exige hoy mayores esfuerzos de coordinación y clarificación de competencias entre los diferentes niveles administrativos. Además, la lógica jerárquica del ejercicio de poder ya no es válida en el actual contexto de interdependencia y poder relacional. En nuestra opinión, sólo desde la transversalidad será posible diseñar y planificar intervenciones estratégicas, integrales y verdaderamente efectivas de lucha contra la exclusión social.

Otro de los elementos fundamentales de la intervención social es, desde nuestro punto de vista, la consideración del conflicto y la diversidad como elementos consustanciales de los procesos sociales. El conflicto no debe ser entendido de forma negativa sino como una importante fuente de creatividad y enriquecimiento social. En este sentido, las políticas públicas no deben pretender evitar y ocultar los conflictos sociales sino aprender a gestionarlos y positivizarlos.
A su vez, partiendo de la idea que la exclusión es un fenómeno multifactorial, es necesario que las acciones contra la exclusión aborden simultáneamente factores diversos que aparecen interrelacionados, y que en muchas ocasiones se retroalimentan. Así pues, las políticas que traten de dar respuesta a la exclusión social han de ser lo más integrales posible, lo que hace necesaria su transversalidad entendida como forma de coordinación flexible de los diferentes actores que intervienen en el territorio, gubernamentales o no, y articulada desde la mayor proximidad posible. Es decir, desde el propio territorio, y desde el ámbito local.

Todos estos retos no son fáciles de afrontar, y menos todavía en un contexto de fragmentación social y cultural, de recorte del Estado del Bienestar, de intensificación y creciente complejidad de los procesos de exclusión social. Un contexto en el que, además, la administración y los agentes locales son los que más a menudo se ven obligados a intervenir sobre problemas enraizados en otros espacios más globales. Sin embargo, y como hemos apuntado ya, el nivel local sigue manteniendo una fuerte capacidad de incidencia sobre estos procesos.

(Artigo recebido em fevereiro de 2005. Versão definitva em março de 2005) 


\section{Referências bibliográficas}

Adelantado, J. Cambios en el estado de bienestar. Barcelona: Icaria, 2000.

Barnes, M. et al. Poverty and social exclusion in Europe. United Kingdom: Edward Elgar, 2002.

BECK, U. La sociedad del riesgo. Barcelona: Paidós, 1998.

Blanco I.; GomÀ, R. Gobiernos locales y redes participativas. Barcelona: Ariel, 2002.

Bommes M.; GedDens, A. Immigration and welfare: Challenging the borders of the welfare state. London: Routledge, 2000.

CAstel, R. Les metamorphoses de la question social. Paris: Gallimard, 1995

EDwards, R.; Glover, J. Risk and citizenship. London: Routledge, 2001.

European Comission. Towards a Europe of Solidarity: Intensifying the Fight Against Social Exclusion, Fostering Integration. COM(92/542), 1992.

Gallego, R., GomÀ, R.; Subirats, J. Políticas sociales y descentralización en España. Madrid: Tecnos-UPF, 2002.

Geddens, M.; Benington, J. Local partnerships and social exclusion in the EU. London:

Routledge, 2002.

Gomà, R.; Subirats, J. Políticas públicas en España. Barcelona: Ariel, 1998.

Goul, J; Jensen, P. Changing labour markets, welfare policies and citizenship. Bristol: The Policy Press, 2002

KICKert, J. et al. Managing complex networks. London: Sage, 1997.

LEVITAs, R. The inclusive society?. London: Macmillan, 1998.

LEWIS, J. Gender, social care and welfare state restructuring in Europe. United Kingdom: Ashgate, 1998.

Pierson, J. Tackling social exclusion. London: Routledge, 2002

Percy Smith, J. Policy responses to social exclusion. London: Open University Press, 2000.

Saraceno, C. Social assistance dynamics in Europe. Bristol: The Policy Press, 2002.

SAINSBURY, D. Gender and welfare states regimes. Oxford: Oxford University Press, 1999.

Subirats, J. (dir.). Un paso más hacia la inclusión social. Plataforma de ONG's de Acción Social. Madrid: Colección Estudios, 2004. 


\section{Resumo - Resumen - Abstract}

\section{Exclusão social e políticas de inclusão. Alguns elementos sobre a realidade européia e espanhola \\ Joan Subirats, Maria Giménez e Anna Obradors}

A globalização impactou fortemente o mercado de trabalho europeu, abrindo caminho para a precarização do trabalho. Enquanto o capital se internacionaliza, o trabalho permanece local e sujeito, em escala crescente, aos fluxos econômicos mundiais. Consolidaram-se, além disso, os padrões da sociedade de consumo, promovendo novas desigualdades sociais e aumentando o risco de exclusão e vulnerabilidade social. O declínio progressivo do industrialismo também afeta as principais bases da estrutura social, tais como os papéis de gênero e as relações familiares. Diferentes redes surgem como chave para a inclusão, especialmente no contexto de crise do Estado de Bem-Estar Social em que se encontra a Europa. A análise da exclusão social deve, assim, levar em conta a evolução das políticas sociais em toda a Europa e sua atual tendência à descentralização e focalização. O objetivo deste artigo é, neste sentido, não somente descrever o fenômeno da exclusão social, mas também lançar idéias para a elaboração de novos critérios para a redefinição das políticas sociais.

Palavras-chave: exclusão social; pobreza; políticas de inclusão

\section{Exclusión social y políticas de inclusión. Algunos elementos sobre la realidad europea y española \\ Joan Subirats, Maria Giménezy Anna Obradors}

La globalización económica ha impactado fuertemente en el mercado laboral europeo, abriendo puertas para el trabajo precario. Mientras el capital se tor na global, el trabajo permanece local y más sumiso a los flujos económicos mundiales. Además, los patrones de la sociedad de consumo se han consolidado, promoviendo nuevas desigualdades sociales y aumentando los riesgos de exclusión y vulnerabilidad social. La caída progresiva del industrialismo también afecta las principales bases de la estructura social como los roles de género o las relaciones familiares. Diferentes redes aparecen como clave para la inclusión, especialmente en el actual contexto de crisis del Estado de Bienestar Social en que se encuentra Europa. Es decir, el análisis de la exclusión social debe tener en cuenta la evolución de las políticas sociales en toda Europa y la tendencia actual de descentralización y focalización de los ser vicios sociales. El objetivo de este artículo es, por lo tanto, no sólo describir el fenómeno de la exclusión social, sino también lanzar ideas para construir nuevos criterios de redefinición de las políticas sociales.

Palabras clave: exclusión social; pobreza; políticas de inclusión 
Social exclusion and inclusion policies. Some elements of the European and Spanish reality

Joan Subirats, Maria Giménez and Anna Obradors

Economic globalisation has had a strong impact over the European labour market, paving the way to the precarious work. While the capital becomes global, work still remains local and more submitted to world economic flows. Moreover, the patterns of consumer society have been consolidated, promoting new social inequalities and increasing the risks of social exclusion. The progressive decline of industrialism also affects the main bases of social structure like gender roles or family's rela tionships. Different networks appear as a key to inclusion, especially in the context of Welfare State crisis, in which Europe is involved. It is to say, the analysis of how the social exclusion scene is set must take into account the critical situation of the social policy all around Europe, and its tendency to decentralization and focalization. It is our purpose not only to describe the social exclusion phenomena but also to provide some ideas to build new criteria for the social policy.

Key words: social exclusion; poverty; inclusion policy

Joan Subirats i Humet

Catedrático de Ciência Política e diretor do Instituto de Governo e Políticas Públicas da Universidade Autônoma de Barcelona (UAB). Cátedra Príncipe de Astúrias na Universidade Georgetown (EUA) no curso 2003-2004. Contato: joan.subirats@uab.es.

Maria Giménez Cencillo

Licenciada em Ciência Política e Ciência da Administração pela UAB. Mestre em Gestão Pública pela Univer sidade Pompeu Fabra, UAB e ESADE. É atualmente coordenadora de pesquisa do Instituto de Governo e Políticas Públicas da UAB. Contato: maria.gimenez@uab.es.

Anna Obradors i Pineda

Licenciada em Sociologia pela UAB e doutoranda em Sociologia pela mesma universidade. É atualmente pesquisadora do Instituto de Governo e Políticas Públicas da UAB. Contato: anna.obradors@uab.es. 


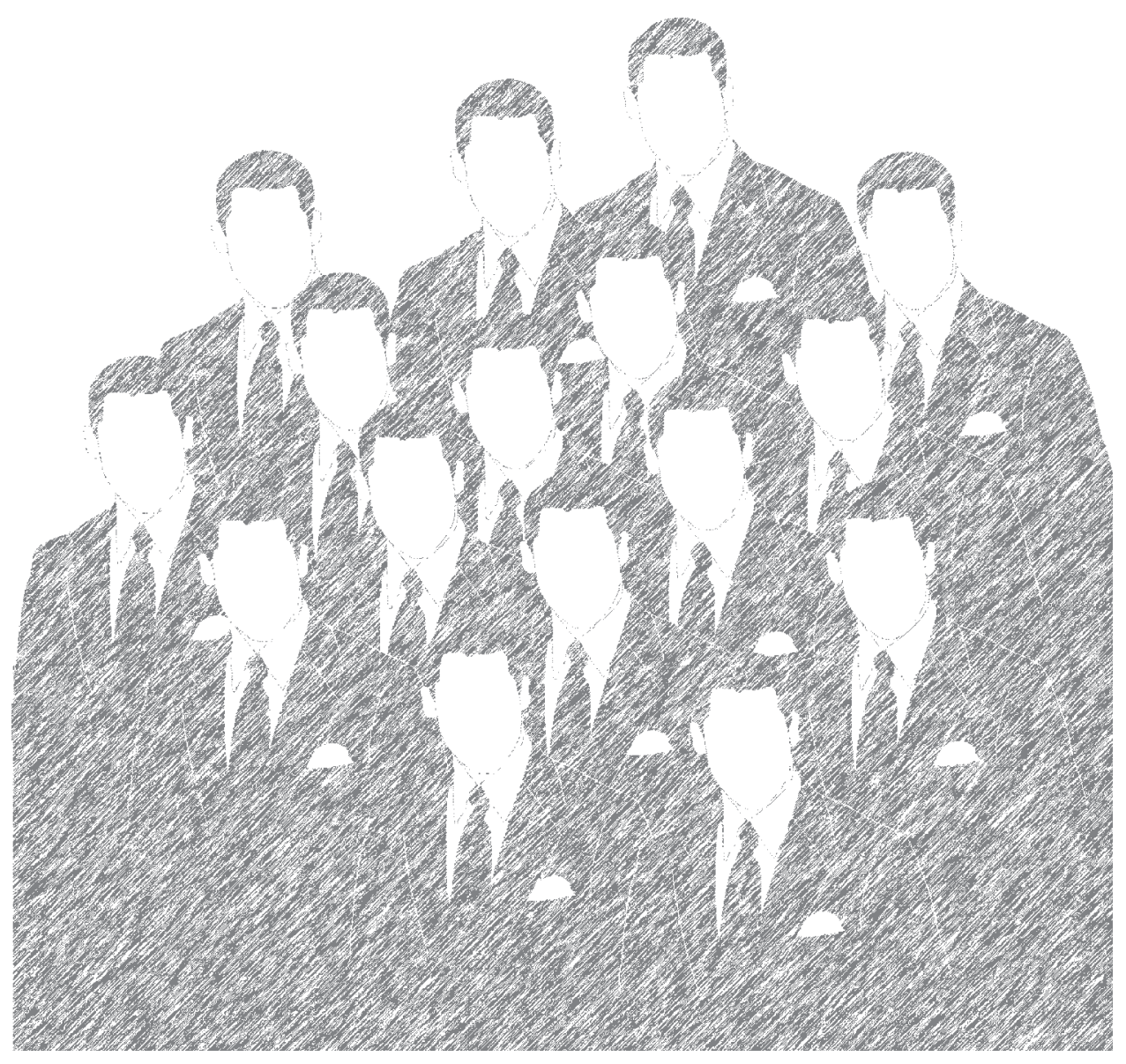

